\title{
Evaluation of Postoperative Delirium: Validity and Reliability of the Nursing Delirium Screening Scale in the Turkish Language
}

\author{
Fadime Çınar ${ }^{a}$ Fatma Eti Aslan ${ }^{b}$ \\ aSabahattin Zaim University, Faculty of Health Sciences, Istanbul, Turkey; ${ }^{\text {b } B a h c ̧ e s ̧ e h i r ~}$ \\ University, Faculty of Health Sciences, Istanbul, Turkey
}

\section{Keywords}

Nursing personnel · Delirium of mixed origin · Reliability · Validity

\begin{abstract}
Purpose: Postoperative delirium is the most well-known form of postoperative cognitive impairment in all patient groups, especially in the elderly. Delirium is a syndrome that causes serious consequences, increasing mortality and morbidity rates and extending the length of hospital stay. The aim of this study was to evaluate the validity and reliability of the Turkish version of the Nursing Delirium Screening Scale (Nu-DESC). Method: One hundred twelve patients who were hospitalized for a surgical operation in the orthopedics, neurosurgery, and general surgery clinic of a state hospital for 3 months were evaluated concurrently (and independently for delirium). Patients were observed by clinical nurses 3 times over a 24-h period. The presence of delirium was diagnosed by 2 neurologists according to DSM-IV criteria. Student's $t$ test, the $\chi^{2}$ test, and the Mann-Whitney $U$ test were used, and construct validity, intrascale factor analysis, interrater reliability, and specificity and sensitivity (ROC) analyses were performed for descriptive analysis. SPSS 25.0 and MedCalc18.6 were used for statistical analysis. Results: Delirium was detected in 28 patients according to the Nu-DESC. The ICC (intraclass correlation) is 0.97 in the $95 \%$ confidence interval from 0.96 to 0.98 for agreement between nurses and neurologists for the total Nu-DESC score. Weighted $\mathrm{k}$ rates were between 0.78 and 0.92 . In the ROC analysis of the Nu-DESC scale, the optimum cutoff value calculated for the 1,344 observations and 112 patients was determined as $>1$ according to the maximum sensitivity and the specific situation. Sensitivity at the cut-off point was 92.27 ; specificity was determined as 92.72. The Youden index was found to be $J=0.845(0<J=0.845<1)$. Conclusion: We believe that Turkish translation of Nu-DESC is valid and reliable for clinicians, nurses, and researchers and will contribute to delirium studies.




\section{Introduction}

Delirium begins with an acute onset of worsening brain function, especially with attention deficit, disorganized thinking, increased or decreased psychomotor activity, and irregularity of the sleep/wake cycle. It is a neuropsychiatric syndrome characterized by changes in consciousness levels [1-3]. Delirium affects patients, their families, and the health system, representing emotional, functionality, and financial burdens [4] and resulting in a prolonged hospital stay, increased mortality and morbidity, and consequently increased hospital costs $[1,5-12]$.

\section{Review of the Literature}

When epidemiological studies in the literature are considered in all patients hospitalized in general, the prevalence of delirium in these patients is $10-30 \%$ [1]. Although there are many factors that cause delirium, elderly patients are more frequently observed among patients undergoing cardiac and hip surgery $[10,13]$. Although delirium can be seen in all age groups, it is reported that the incidence increases after 60 years of age, and it is more common in patients aged over 80 years [14]. It is reportedly seen in $41-60 \%$ of patients after total knee replacement surgery and in $40-60 \%$ of patients after femoral neck fracture surgery [15].

According to psychomotor behavior changes, delirium is classified as hypoactive, hyperactive, or mixed type. In hyperactive delirium, the patient is hypersensitive to stimuli and psychomotor activity is increased. In hypoactive delirium, sensitivity and psychomotor activity are decreased. A patient may be both hyperactive and hypoactive during the day, which may also be called a mixed type $[3,6,12,16,17]$. If the delirium cannot be diagnosed early and the underlying cause is incurable, it can result in death [18]. For this reason, it is very important that delirium be diagnosed early and the underlying cause treated. Although it is clinically relevant, delirium is often unrecognized or misdiagnosed by health professionals because its symptoms are not well recognized or it is confused with diseases such as dementia and depression [6, 19]. In the literature, it has been reported that only $40 \%$ of nurses, doctors, and intensive care workers regularly evaluate the patient in terms of delirium [16].

The Nursing Delirium Screening Scale (Nu-DESC) is the gold standard for the diagnosis of delirium. All other psychiatric diagnoses are classified according to the criteria of the international guideline, i.e., the DSM-V Diagnostic and Statistical Manual for Mental Disorders. However, diagnosis of delirium using these criteria requires both time consuming and comprehensive training. A diagnosis of delirium can be made only via a clinical evaluation. Therefore, in addition to DSM-V criteria, some diagnostic scales are recommended for the diagnosis of delirium and to determine the severity of the findings $[20,21]$. Considering the prevalence and negative results of delirium, specialized strategies in delirium management, diagnosis, treatment, and care are important $[6,12]$. Spronk et al. [22] found that nurses and doctors did not adequately diagnose delirium and they did not use adequate measuring instruments to make a diagnosis. In the study of Hipp and Ely [23] it was determined that delirium was overlooked and could not be treated. Ely et al. [16] found that only $16 \%$ of health care workers used a specific delirium measurement form in a different study with 912 intensive care workers.

There are various scales that measure the signs and severity of delirium [24]. One of them is the Nu-DESC. Gaudreauete et al. [25] developed the Nu-DESC, which is designed for nurses who work in clinics with inpatients are and therefore will witness the characteristic effects of delirium. The Nu-DESC is a scale that evaluates the severity of the 5 delirium characteristics 
on a scale of 0 (not available) to 2 (severe) and takes only 1-2 min to complete, based on the nurses' observations of the patient's behavior during their shifts. The Nu-DESC is short and simple and easy to use for nurses [25]. Use of a scale in accordance with scientific principles is only possible if it has been proven to be standard, valid, and reliable in the form adapted to the target population. Even the most widely used, well-known scales are studied in different languages, and different validity and reliability results can be reported. Therefore, this study aimed to validate the reliability and validity of the Turkish version of the Nu-DESC.

\section{Methods}

\section{Study Design}

Delirium is a common neuropsychiatric disorder. The Nu-DESC, which is widely used for the diagnosis of delirium, is a well-validated scale that can be easily used by nurses. This study was carried out to translate the English Nu-DESC and adapt it to the Turkish language, to make it available to patients undergoing surgery, and to make it available for validity and reliability studies. The research was conducted in a public hospital operating in the province of Istanbul, and it is observational and of the methodological type.

\section{Setting and Sample}

The study population consisted of patients who were admitted to the orthopedics, neurosurgery, and general surgery clinics between June 2018 and September 2018 when this study was conducted. No sampling method was used and all of the universe was included in this study. One hundred sixty-seven samples were observed for the diagnosis of Nu-DESC delirium. However, due to incomplete and inaccurate completion of the items in the scale by the observers, the scale data of 55 samples were excluded. One hundred twelve individuals who agreed to participate in this study on the basis of volunteerism constituted the sample of this study. In the literature, it has been stated that a sample size 5-10 times the number of items in the scale is sufficient $[26,27]$. The sample size is more than 10 times the number of items in the scale. The exclusion criteria were as follows: ASA score III; age under 18 years; a preoperative delirium history; a history of neurological or mental illness or sedative or antidepressant use; glucocorticoid use; a language barrier; a serious hearing or visual impairment; and alcohol or drug addiction. In addition, patients were included in this study at least $24 \mathrm{~h}$ before the operation and the participants were observed using the scale until discharge.

\section{Ethical Considerations}

This study was approved by the Institutional Review Board of the Sabahattin Zaim University (Approval No. 3,517/2018) and permission from the management of the hospital was obtained. After participation was stated on a voluntary basis, written and oral informed consent of the participants was obtained. Before the research process, permission was obtained from the researchers who developed the scale for the use of the Nu-DESC and the study of validity and reliability of the scale for Turkish society. Health workers in the clinics included in this study were informed about the purpose, content, and method of this study.

\section{Measurements/Instruments}

Individual Information Form

The questionnaire consisted of questions about age, gender, ASA physical status, anesthesia type, the duration of anesthesia, the duration of surgery, the length of intensive care unit stay, and the duration of hospital stay. 
Nursing Delirium Screening Scale

Gaudreau et al. [25] developed the Nu-DESC. The psychometric properties of Nu-DESC were determined using the categories of the Confusion Evaluation (CAM) performed by nurses and psychiatrists. In this study, the Nu- DESC was psychometrically validated and had $85.7 \%$ sensitivity and $86.8 \%$ specificity. It was concluded that the Nu-DESC is promising for clinical use in in-hospital research environments and as an instrument that can be used in research. The Nu-DESC is a 5 -item observation scale that can be completed quickly. Orientation disorder, inappropriate behavior, inappropriate communication, illusion-hallucination, and psychomotor deceleration are the items of delirium screening scale. Each item is evaluated on a 3-point Likert (from 0 to 3 ). The total score is obtained by adding the values of the items, and the highest score is 10 . If the total score is 2 and above, the diagnosed of delirium is made. It takes less than 2 min to complete and is intended for use by nurses.

Intensive Care Delirium Screening Checklist

The Intensive Care Delirium Screening Checklist (ICDSC) is an 8-item checklist that evaluates delirium symptoms over 8-24 h. ICDSC assesses the level of consciousness, attention deficit, disorientation, hallucinations, delusions, psychoses, psychomotor agitation or retardation, inappropriate speech or affect, sleep/vigil loop disorders, and symptom fluctuations. If the symptoms on the list are not present in the patient, no score is given for that symptom on the list; an evaluation score $>4$ indicates the presence of delirium. The ICDSC is easy to implement and can be completed in a short time, and the scoring system and interpretation of scores are practical [28].

\section{Data Collection}

In this study, the Individual Information Form, the Nu-DESC, and the Intensive Screen Control-ICDSC were used. The data were observed by nurses working independently in clinics and the diagnoses of all of the cases were collected by 2 specialist neurologists, one in the day shift and one in the night shift.

\section{Evaluation of Delirium}

Daily delirium assessments of the patients were performed using the Nu-DESC by 2 neurologists and trained nurses. Screening took place every day for $24 \mathrm{~h}$. Since delirium was frequently seen on the second or third postoperative day [5], patients were observed for the diagnosis of delirium in total 1,344 times, one day before surgery, up to 3 days postoperatively, three times daily observation was performed and recorded. The presence of delirium was determined by clinical nurses and 2 neurologists using DSM-IV criteria as the gold standard and with the Nu-DESC and the ICDSC. The researchers who made the tests were allowed to be blind to each other. The delirium screening was considered as a separate criterion of this study because the participants who were treated in the clinic first were transferred to the intensive care unit after the operation. Patients with intensive care transfer were excluded from this study.

\section{Language Adaptation of the Scale}

Both the group translation technique and the back translation technique were used for language adaptation of the scale. For the group translation, 5 people whose native language was Turkish and who studied English did the translation into English. Afterward, the researchers and a linguist in the field of English evaluated the text and the scale items were agreed upon. The back translation was done an American expert living in Turkey. These translations were compared with the original language by 2 faculty members and the nonoriginal articles were reviewed in terms of Turkish. 
Validity and Reliability Study

The content validity of the scale was assessed using the Lawshe method to assess the validity of the scale after the language adaptation. For the validity of the content, the 5 -item scale was communicated to 2 faculty members from the Department of Internal Medicine Nursing, 2 faculty members from the Human and Society Department, and 2 surgical nurses and 1 measurement and evaluation specialist. A form was prepared for expert opinions. In that form, sent via e-mail, the options were: (1) each item measures the targeted structure, (2) the substance is related to the structure but is unnecessary, or (3) the substance does not measure the targeted structure. Scope validity ratios were obtained by gathering the opinions of the experts about any item in this method [26, 27].

Reliability Studies

The internal consistency coefficients and interrater $\kappa$ (reliability compliance) coefficients were examined. In addition, item total correlation coefficients were examined for 5 items in the scale. The invariance of the scale according to time (test-retest) was evaluated. A subgroup of 34 patients was screened separately by 2 different nurses in less than $60 \mathrm{~min}$ in order to evaluate the intercept reliability for each delirium score. For each score, each delirium score was recorded for 10 different delirium screening events.

\section{Data Analysis}

Student's $t$ test for descriptive analysis, the $\chi^{2}$ test, the Mann-Whitney $U$ test for intergroup comparisons, construct validity, intrascale factor analysis, interrater reliability, compliance of the Nu-DESC with the ICDSC, internal consistency analysis, specificity and sensitivity, and diagnostic accuracy (ROC) analysis were performed for various cut-off values of delirium scores. While evaluating the findings of this study, SPSS 25.0 (SPSS, Inc., Chicago, IL, USA) and MedCalc18.6 (http://www.statsdirect.com, StatsDirect Ltd., UK), and MedCalc version 18.6 ( 64 bit $^{\odot}$, Mariakerke, Belgium) were used. $p<0.005$ was considered statistically significant. One of the methods used in the comparison of 2 diagnostic tests is the Youden index (J). What diagnostic test has a higher diagnostic power? The answer to the question was searched. $\mathrm{J}=1-(\mathrm{FN}+\mathrm{FP})$, where FN = false-negative rate and FP = false-positive rate. The following considerations are important: (1) the value of J varies from -1 to +1 ; (2) if it is smaller than zero, it indicates that the test has no diagnostic power; and (3) when comparing the strengths of 2 diagnostic tests it can be said that the greater the J value is, roughly, the more effective the diagnostic test is.

\section{Results}

\section{Demographic Characteristics of the Participants}

Of the 112 patients, 55 (49.1\%) were male and 57 (50.9\%) were female. The rate of males was significantly higher in delirium cases than in patients without delirium $(p=0.003)$. The ratio of patients who had an ASA score $>2(p=0.004)$, the length of anesthesia time $(p=$ $0.006)$, the length of surgical operation time $(p=0.026)$, the mean age $(p=0.37)$, and the length of hospitalization ( $p=0.005)$ were significantly greater than in the nondelirium group (Table 1).

\section{Scope Validity and Substance Analysis of the Scale}

According to the Lawshe method, the validity values of all of the items were above the lower limit of 0.59 for 11 experts. It was found to be between 0.64 and 1.00 . Item analysis of the scale was performed and item total score correlations of 5 items were analyzed. The item 
Table 1. Demographics and Nu-DESC scores of hospitalized patients grouped by diagnosis

\begin{tabular}{lccc}
\hline Item & Delirium & & \\
\hline Males & $19(67.9)$ & No delirium $^{\mathrm{b}}$ & \\
Females & $9(32.1)$ & $36(42.9)$ & $0.003^{\mathrm{d}}$ \\
Orthopedics clinic & $15(53.5)$ & $48(57.1)$ & \\
Brain surgery clinic & $9(32.2)$ & $34(40.5)$ & $0.043^{\mathrm{d}}$ \\
General surgery & $414.3)$ & $22(26.2)$ & \\
ASA score >2 & $22(78.5)$ & $15(17.9)$ & \\
Age, years & $64.75 \pm 18.55$ & $45.75 \pm 14.55$ & $0.004^{\mathrm{d}}$ \\
Length of hospitalization, days & $12.46 \pm 3.78$ & $5.37 \pm 3.22$ & $0.037^{\mathrm{c}}$ \\
Anesthesia duration, min & $101.32 \pm 35.17$ & $67.54 \pm 11.13$ & $0.005^{\mathrm{c}}$ \\
Surgery duration, min & $89.97 \pm 15.39$ & $44.27 \pm 14.23$ & $0.006^{\mathrm{c}}$ \\
\hline
\end{tabular}

Values are presented as numbers (\%) or means \pm SD. ${ }^{\mathrm{a}} n=28 .{ }^{\mathrm{b}} n=41 .{ }^{\mathrm{c}}$ Student's $t$ test $(p<0.001)$. ${ }^{\mathrm{d}} \chi^{2}$ test $(p<0.005)$

Table 2. Nu-DESC dimensions

\begin{tabular}{llll}
\hline Dimension & Delirium & Non delirium & $p$ value \\
\hline Orientation disorder & $2(1-2)$ & $1(0-1)$ & $<0.001$ \\
Improper conduct & $1(0-2)$ & $0(0-1)$ & $<0.001$ \\
Inappropriate communication & $2(0-2)$ & $0(0-1)$ & $<0.001$ \\
Illusions/hallucinations & $2(1-2)$ & $0(0-0)$ & $<0.001$ \\
Psychomotor deceleration & $2(0-2)$ & $0(0-1)$ & $<0.001$ \\
Delirium (total score $\geq 2$ points) & $7(2-10)$ & $2(1-4)$ & $<0.001$ \\
\hline
\end{tabular}

Values are means (range). All values are significantly different between groups at $p<0.001$. MannWhitney U test, $p<0.001$.

total correlation coefficients of the scale were found to be $r=0.48$ and 0.73 , positive, and statistically significant. In order to test the internal consistency within the scope of reliability, Cronbach $\alpha$ values were examined. The total Cronbach $\alpha$ value of the scale was determined to be 0.95 . The relationship between the scale and its subdimensions was evaluated by Spearman-Brown correlation analysis and a statistically significant $(p<0.001$ and $p<0.005)$ positive correlation was found between the total score and all subdimension scores of the scale. For time invariance (test-retest), the application was repeated in 34 people for 2 weeks. Pearson's correlation was found to be $0.76(p=0.000)$.

There was a statistically significant difference $(p<0.001)$ between patients who had delirium and those who had averages of all of the dimensions of the Nu-DESC (Table 2).

For all patient evaluations using the Nu-DESC, the closure rates between nurses and specialist neurologists were between 0.78 and $0.92(>0.96)$ (Table 3). The ICC (intraclass correlation) is 0.97 in the $95 \%$ confidence interval from 0.96 to 0.98 for agreement between nurses and neurologists for the total Nu-DESC score.

Delirium Diagnostic Findings with the ICDSC and the Nu-DESC

The subjects included in this study were observed for the diagnosis of delirium 3 times a day. Patients were observed 1 day before surgery and 3 days after surgery. A total of 1,344 observations were performed. Five (false-negative rate: $17.8 \%$ ) patients were found to have 
Table 3. Cronbach's $\alpha$ coefficient of the Nu-DESC and $\kappa$ adaptation rates between nurses and neurology specialist doctors

\begin{tabular}{llll}
\hline Nu-DESC dimension & $\begin{array}{l}\text { Cronbach's } \alpha \\
\text { coefficient }\end{array}$ & $\begin{array}{l}\text { Weighted } \kappa \\
(95 \% \mathrm{CI})\end{array}$ & $\begin{array}{l}\text { Compliance rate } \\
(95 \% \mathrm{CI})\end{array}$ \\
\hline Orientation disorder & 0.93 & $0.87(0.73-1.00)$ & $0.97(0.87-0.98)$ \\
Improper conduct & 0.88 & $0.89(0.76-1.00)$ & $0.97(0.88-0.99)$ \\
Inappropriate communication & 0.96 & $0.89(0.76-1.00)$ & $0.97(0.88-0.99)$ \\
Illusions/hallucinations & 0.83 & $0.92(0.84-1.00)$ & $0.98(0.90-1.00)$ \\
Psychomotor deceleration & 0.94 & $0.78(0.40-1.00)$ & $0.96(0.90-1.00)$ \\
Delirium (total score $\geq 2$ points) & 0.95 & $1.00(1.00-1.00)$ & $0.97(0.90-1.00)$ \\
\hline
\end{tabular}

The total number of subjects is 112 .

Table 4. Nu-DESC And ICDSC definitive diagnostic findings

\begin{tabular}{lcccc}
\hline & ICDSC & Nu-DESC & $\chi^{2}$ & $p$ value \\
\hline Nondelirium & $91(81.2)$ & $84(75)$ & 116.2 & 0.452 \\
Delirium & $21(18.8)$ & $28(25)$ & 116.2 & 0.001 \\
Hypoactive delirium & $10(47.6)$ & $12(48.9)$ & 113.7 & 0.523 \\
Hyperactive delirium & $11(52.3)$ & $6(21.4)$ & 109.8 & 0.624 \\
Mixed delirium & $0(0)$ & $10(35.7)$ & 111.8 & 0.003 \\
\hline Total & $112(100)$ & $112(100)$ & & \\
\hline
\end{tabular}

Values are presented as numbers (\%). $p<0.001$ and $p>0.005$

no delirium. According to ICDSC, delirium was present in 4 patients who were not diagnosed with delirium by Nu-DESC (false-positive rate: $5.8 \%)(n=112)$. The rates of detecting hypoactive and hyperactive delirium-type cases were similar in patients with Nu-DESC $(n=28)$ and ICDSC $(n=23)$. However, mixed delirium was detected only with Nu-DESC. A significant relationship was found between Nu-DESC scale and ICDSC $\left(\chi^{2}=116.2, p=0.001\right)(p<0.001)$ (Table 4).

\section{Delirium Scores using ROC Analysis}

With ROC analysis comparing the delirium and nondelirium groups for Nu-DESC scores, sensitivity and specificity for various cut-off values for the Nu-DESC were determined. With the ROC analysis, the optimum cut-off value calculated for 1,344 observations and 112 patients according to the maximum sensitivity and special case was determined to be $>1$. The sensitivity at the cut-off point was 92.27 ; specificity was determined to be 92.72 . The Youden index was found to be $\mathrm{J}=0.845(0<0.845<1)$. The areas under the ROC were found to be statistically significant $(p<0.05)$. The area under the curve was 0.86 for the ICDSC test (95\% CI 0.83-0.93), and the calculated optimum cut-off value was $>4$. For the Nu-DESC scale (area under the curve) the value was 0.95 and $95 \%$ CI was $0.93-0.96(p<0.001)$. The expected mortality rate for the delirium score was calculated as $25.09 \%$ (Table 5).

When the average application time of the ICDSC scale with the Nu-DESC scale was examined, it was seen that the Nu-DESC scale could be applied in an average of $2.13 \pm 0.05$ min and the ICDSC test could be applied in a mean of $3.15 \pm 0.18 \mathrm{~min}$. It was found that the $\mathrm{Nu}$-DESC could be applied in a shorter time $(p<0.001)$. 
Çınar and Eti Aslan: Validity and Reliability of the Nu-DESC in the Turkish Language

Table 5. Meaning of delirium ROC curve and delirium score criteria according to sensitivity and specificity values

\begin{tabular}{lllllllll}
\hline & AUC & $95 \%$ CI & $p$ & Criteria & Sensitivity & $95 \%$ CI & Specificity & $95 \%$ CI \\
\hline ICDSC & 0.86 & $0.80-0.91$ & $<0.001$ & $>1$ & 92.27 & $87.82-95.52$ & 92.72 & $90.43-94.61$ \\
Nu-DESC & 0.95 & $0.93-0.96$ & $<0.001$ & $>4$ & 78.14 & $60.52-91.78$ & 89.68 & $86.72-91.62$ \\
$p$ value (Nu DESC and ICDSC) & & & & & 0.017 & & 0.103 & $98.84-100.00$ \\
\hline
\end{tabular}

\section{Discussion}

Delirium is one of the most common acute disorders in adult patients in hospitals [1]. It can be diagnosed at an early stage but its development cannot be avoided. Nurses who have one-on-one and continuous communication with the patients and who have the responsibility of closely monitoring them can take the necessary measures to realize delirium in the early period and prevent its progression [6]. Many scales have been developed for the evaluation of delirium [25]. However, there are no sufficiently understandable scales that do not require much time from the nurses, especially in surgical clinics. The Nu-DESC is a tool with a high validity and reliability that nurses can use in clinics. Commonly used for the diagnosis and determination of the severity of delirium, it has been translated into many languages. The aim of this study was to investigate the validity and reliability of the Nu-DESC scale in Turkish

In our study, delirium screening tests were applied in patients who had stayed in surgery clinics for longer than $48 \mathrm{~h}$. It was reported that delirium started on average between 2 and 3 days after admission and surgery and lasted for 3-4 days, although it could last up to 60 days [29, 32].

In the literature, it is stated that male gender is one of the factors that increase the tendency of delirium [32,33]. In our study, the 19 males $(67.9 \%)$ and 9 women $(32.1 \%)$ developed delirium, and there was a statistically significant difference $(p<0.05)$ and our results were similar to the literature. In this study, the ASA score was $>2$ in $15 \%$ of the 60 patients with delirium and this was similar to results reported in the literature [34]. The mean duration of hospital stay was 15 days in patients who developed delirium and 7 days in those who did not. In our study, the mean length of hospital stay for delirium patients was $12.46 \pm 3.78$ days and a statistically significant difference was found [3].

Validity and reliability and 2 important criteria that are expected to be fulfilled by all scales used in research. The extent to which the measuring instrument is suitable and its degree of measurement are defined as the desired condition or the property to be measured. Reliability is that the measurement tool has the same degree of consistency as the item to be measured, or that the answers of the respondents are consistent with each other. Thus, the measurement tool can be accepted as valid if the desired property is measured by the measuring instrument [35].

The internal consistency analysis was performed to determine the homogeneity of the scale while testing the reliability of the Nu-DESC scale. In the analysis of internal consistency, item-total score correlation and Cronbach's $\alpha$ values were taken into consideration. The itemtotal score correlation values of the scale were found to be positive $(r=0.48-0.73)$ for 5 items. In the literature, it is emphasized that this value should be $r=0.30$ and above [36].

If the Cronbach $\alpha$ coefficient is high in the literature, it is emphasized that the reliability of the scale is high. The value of this layer at $0.60-0.80$ is deemed to be reliable, and at $0.80-$ 1.00 it is reported to have a high level of reliability [36]. This result shows that the scale is highly reliable. The Nu-DESC was found to be statistically significant $(p<0.001)$ between the subjects with delusions and the average of the items of all sizes. There was a statistically significant difference $(p<0.001)$ between the averages and those without delirium. 
The Nu-DESC also uses 24-h nurse observations as in the ICDSC. The disorientation, inappropriate behavior, inappropriate communication, illusion/hallucinations, and psychomotor retardation in the Nu-DESC are also included in the ICDSC, but they are evaluated with different scores. The Nu-DESC found delirium in 28 patients $(25 \%)$, while the ICDSC found delirium in 23 patients (20.53\%). The patients included in the study were evaluated for the diagnosis of delirium by observing 3 times a day for one day before surgery and for 3 days after surgery. A total of 1,344 observations were performed. According to the ICDSC, 5 patients had no delirium (false-negative rate: $17.8 \%$ ), and 4 patients had delirium (false-positive rate: $5.8 \%)$ among the total cases $(n=112)$. The rates of detecting hypoactive and hyperactive delirium-type cases were similar in patients with Nu-DESC $(n=28)$ and ICDSC $(n=23)$. However, mixed delirium was detected only with Nu-DESC $(p<0.001)$. These results were consistent with the literature [37-40].

ROC analysis comparing the delirium and nondelirium groups based on Nu-DESC scores found sensitivity and specificity for various cut-off values for the Nu-DESC. Based on the ROC analysis, 1,344 observations (according to the maximum sensitivity and the specificity) were made, and the optimal cut-off value calculated for the Nu-DESC in 112 patients was >1; it was $>4$ for the ICDSC. The sensitivity at the cut-off point was 92.27 , and the specificity was 92.72 . The Youden index was $0.845(0<0.845<1)$. Areas under ROC curves were found to be statistically significant $(p<0.05)$. The AUC (area under the ROC curve) for the ICDSC test is between 0.86 and $0.83-0.93$ for the $95 \%$ confidence interval. The AUC value for the Nu-DESC scale was between 0.95 and $0.93-0.96$ for the 95\% confidence interval $(p<0.001)$. The expected mortality rate for the delirium score was calculated as $25.09 \%$. These results were consistent with the literature [25, 27, 41-44].

This study shows that the Turkish version of the Nu-DESC is an accurate and reliable tool for evaluating delirium in patients who have undergone surgery. It also shows that nurses and neurologists can quickly and accurately implement the Nu-DESC. The scale shows a high sensitivity and a high specificity, which are particularly important for a screening/monitoring tool and not particularly different from the properties of the ICDSC. The Nu-DESC has values similar to those in other published studies [25, 27, 41-44]. It is in line with nursing studies evaluating the impact of education efforts focusing on use [17].

\section{Strengths and Limitations}

The Nu-DESC is a delirium screening instrument that can be easily integrated into routine care and clinical practice. Future studies investigating delirium epidemiology, risk factors, and treatment interventions could benefit from the use of the Nu-DESC. Most of all, proper and systematic attention to the cognitive/behavioral status of hospitalized patients through the $\mathrm{Nu}$-DESC could greatly improve global psychiatric patient care. The lack of DSM-5 validation could be stated as a limitation of our study. However, we did not choose to compare the Nu-DESC to DSM-IV or the DSM-5 criteria because an isolated comparison between the $\mathrm{Nu}-\mathrm{DESC}$ and the "new" DSM-5 criteria did not seem meaningful (other Nu-DESC translations and delirium tools where only compared to the DSM-IV) and due to the fact that the whole delirium focus has shifted significantly with the progression of delirium gold standards from the DSM-IV to the DSM-5. Now there is only a 30\% overlap between the DSM-IV and the DSM-5 in delirium diagnosis using the strict definition. Additionally, the Nu-DESC has been abundantly validated in comparison to the DSM-IV in numerous different languages and settings

\section{Implications for Practice}

The Nu-DESC is easy to use, time efficient, and accurate and could lead to prompt delirium recognition and treatment. It shows promise as a useful concomitant delirium research tool, allowing continuous screening, symptom monitoring, and severity rating. 


\section{Conclusion}

The Turkish version of the Nu-DESC was found to be a highly reliable and valid tool for measuring delirium severity and diagnosing delirium in Turkish medical settings. It was found to be very good in terms of intermediate reliability, substance-substance comparison, and delirium diagnosis between physicians and nurses. The psychometric properties of the $\mathrm{Nu}$-DESC were similar to those of the ICDSC, but the low number of Nu-DESC items resulted in a faster administration than the ICDSC. For this reason, the Nu-DESC is promising as a precise diagnostic tool with a high specificity to evaluate delirium not only as a high-precision screening and monitoring tool but also in surgical care environments. Delirium is a serious problem that is common in patients after surgery and cannot be diagnosed or treated, and it increases morbidity and mortality. The causes, diagnosis, clinical features, and results of delirium should be well known. The development of scales for the diagnosis of delirium, training of nurses in this subject and the addition of these screening methods to daily routine are essential.

\section{Statement of Ethics}

This research was conducted according to Helsinki Declaration criteria.

\section{Disclosure Statement}

The authors declare no conflicts of interest.

\section{Funding Sources}

No financial support (funding, stocks, patents, employment, honoraria, or royalties) from any institution was received for this study.

\section{Author Contributions}

Conceptualization, validation, review and editing, and funding acquisition: F.Ç. and F.E.A. Methodology, software, formal analysis, investigation, resources, data curation, preparation of the original draft, visualization, supervision, and project administration: F.Ç.

\section{References}

1 Alexander E. Delirium in the intensive care unit: medications as risk factors. Crit Care Nurse. 2009 Feb;29(1): 85-7.

2 Connor D, English W. Delirium in critical care. ATOTW. 2011;232:1-13.

3 Marcantonio ER. In the clinic: delirium. Ann Intern Med. 2011 Jun;154(11):1-16.

4 Wiesenfeld L. Delirium: the ADVISE approach and tips from the frontlines. Geriatrics. 2008 May;63(5):28-31.

5 Chang YL, Tsai YF, Lin PJ, Chen MC, Liu CY. Prevalence and risk factors for postoperative delirium in a cardiovascular intensive care unit. Am J Crit Care. 2008 Nov;17(6):567-75.

6 Wells LG. Why don't intensive care nurses perform routine delirium assessment? A discussion of the literature. Aust Crit Care. 2012 Aug;25(3):157-61.

7 Rudolph JL, Jones RN, Levkoff SE, Rockett C, Inouye SK, Sellke FW, et al. Derivation and validation of a preoperative prediction rule for delirium after cardiac surgery. Circulation. 2009 Jan;119(2):229-36.

8 Morandi A, Jackson JC, Ely EW. Delirium in the intensive care unit. Int Rev Psychiatry. 2009 Feb;21(1):43-58.

9 Koster S, Hensens AG, Schuurmans MJ, van der Palen J. Risk factors of delirium after cardiac surgery: a systematic review. Eur J Cardiovasc Nurs. 2011 Dec;10(4):197-204. 
10 Van Rompaey B, Schuurmans MJ, Shortridge-Baggett LM, Truijen S, Bossaert L. Risk factors for intensive care delirium: a systematic review. Intensive Crit Care Nurs. 2008 Apr;24(2):98-107.

11 Kamholz B. Update on delirium: diagnosis, management, and pathophysiology. Psychiatr Ann. 2010;40(1): $52-62$.

12 Kristiansen S, Konradsen H, Beck M. Nurses' experiences of caring for older patients afflicted by delirium in a neurological department. J Clin Nurs. 2019 Mar;28(5-6):920-30.

13 Siddiqi N, House AO, Holmes JD. Occurrence and outcome of delirium in medical in-patients: a systematic literature review. Age Ageing. 2006 Jul;35(4):350-64.

14 Fong TG, Tulebaev SR, Inouye SK. Delirium in elderly adults: diagnosis, prevention and treatment. Nat Rev Neurol. 2009 Apr;5(4):210-20.

15 Caraceni A, Grassi L. Delirium: acute confusional states in palliative medicine. 2nd ed. Oxford: Oxford University Press; 2011. pp. 1-304.

16 Ely EW, Stephens RK, Jackson JC, Thomason JW, Truman B, Gordon S, et al. Current opinions regarding the importance, diagnosis, and management of delirium in the intensive care unit: a survey of 912 healthcare professionals. Crit Care Med. 2004 Jan;32(1):106-12.

17 Devlin JW, Fong JJ, Howard EP, Skrobik Y, McCoy N, Yasuda C, et al. Assessment of delirium in the intensive care unit: nursing practices and perceptions. Am J Crit Care. 2008 Nov;17(6):555-65.

18 Eeles EM, Hubbard RE, White SV, O'Mahony MS, Savva GM, Bayer AJ. Hospital use, institutionalisation and mortality associated with delirium. Age Ageing. 2010 Jul;39(4):470-5.

19 Banerjee A, Vasilevskis EE, Pandharipande P. Clinical review strategies to improve delirium assessment practices in the intensive care unit. Journal of Clinical Outcomes Management. 2010;17(10):459.

20 Brummel NE, Vasilevskis EE, Han JH, Boehm L, Pun BT, Ely EW. Implementing delirium screening in the ICU: secrets to success. Crit Care Med. 2013 Sep;41(9):2196-208.

21 Cinar MA, Ozmenler KN, Ozsahin A, Trzepacz PT. Reliability and validity of the Turkish translation of the Delirium Rating Scale-Revised-98. Dusunen Adam. 2014;27(3):186-93.

22 Spronk PE, Riekerk B, Hofhuis J, Rommes JH. Occurrence of delirium is severely underestimated in the ICU during daily care. Intensive Care Med. 2009 Jul;35(7):1276-80.

23 Hipp DM, Ely EW. Pharmacological and nonpharmacological management of delirium in critically ill patients. Neurotherapeutics. 2012 Jan;9(1):158-75.

24 Kırpınar İ. Assessing and managing delirium in surgical and internal medicine: an overview. Bezmialem Sci. 2016;4(3):113-22.

25 Gaudreau JD, Gagnon P, Harel F, Tremblay A, Roy MA. Fast, systematic, and continuous delirium assessment in hospitalized patients: the nursing delirium screening scale. J Pain Symptom Manage. 2005 Apr;29(4):36875.

26 Martínez-Mesa J, González-Chica DA, Bastos JL, Bonamigo RR, Duquia RP. Sample size: how many participants do I need in my research? An Bras Dermatol. 2014 Jul-Aug;89(4):609-15.

27 Leung L. Validity, reliability, and generalizability in qualitative research. J Family Med Prim Care. 2015 Jul-Sep; 4(3):324-7.

28 Mohajan HK. Two criteria for good measurements in research: validity and reliability. Ann Spiru Haret Univ. 2017;17(4):59.

29 Freter SH, Dunbar MJ, MacLeod H, Morrison M, MacKnight C, Rockwood K. Predicting post-operative delirium in elective orthopaedic patients: the Delirium Elderly At-Risk (DEAR) instrument. Age Ageing. 2005 Mar; 34(2):169-71.

30 Ebell MH. Predicting delirium in hospitalized older patients. Am Fam Physician. 2007 Nov;76(10):1527-9.

31 Barr J, Fraser GL, Puntillo K, Ely EW, Gélinas C, Dasta JF, et al.; American College of Critical Care Medicine. Clinical practice guidelines for the management of pain, agitation, and delirium in adult patients in the intensive care unit. Crit Care Med. 2013 Jan;41(1):263-306.

32 Pappachan B, Agrawal R. Post-operative delirium. J Maxillofac Oral Surg. 2019 Mar;18(1):157-58.

33 Ahmed S, Leurent B, Sampson EL. Risk factors for incident delirium among older people in acute hospital medical units: a systematic review and meta-analysis. Age Ageing. 2014 May;43(3):326-33.

34 Kyziridis T C. Post-operative delirium after hip fracture treatment: a review of the current literature. Psychosoc Med. 2006 Feb 8;3:Doc01.

35 Flora DB, Flake JK.The purpose and practice of exploratory and confirmatory factor analysis in psychological research: decisions for scale development and validation. Can J Behav Sci. 2017;49(2):78.

36 Heale R, Twycross A. Validity and reliability in quantitative studies. Evid Based Nurs. 2015 Jul;18(3):66-7.

37 Minden SL, Carbone LA, Barsky A, Borus JF, Fife A, Fricchione GL, et al. Predictors and outcomes of delirium. Gen Hosp Psychiatry. 2005 May-Jun;27(3):209-14.

38 Norkiene I, Ringaitiene D, Misiuriene I, Samalavicius R, Bubulis R, Baublys A, et al. Incidence and precipitating factors of delirium after coronary artery bypass grafting. Scand Cardiovasc J. 2007 Jun;41(3):180-5.

39 Bickel H, Gradinger R, Kochs E, Förstl H. High risk of cognitive and functional decline after postoperative delirium. A three-year prospective study. Dement Geriatr Cogn Disord. 2008;26(1):26-31.

40 McAlpine JN, Hodgson EJ, Abramowitz S, Richman SM, Su Y, Kelly MG, et al. The incidence and risk factors associated with postoperative delirium in geriatric patients undergoing surgery for suspected gynecologic malignancies. Gynecol Oncol. 2008 May;109(2):296-302. 
41 Radtke FM, Franck M, Schneider M, Luetz A, Seeling M, Heinz A, et al. Comparison of three scores to screen for delirium in the recovery room. Br J Anaesth. 2008 Sep;101(3):338-43.

42 Radtke FM, Franck M, Schust S, Boehme L, Pascher A, Bail HJ, et al. A comparison of three scores to screen for delirium on the surgical ward. World J Surg. 2010 Mar;34(3):487-94.

43 Neufeld KJ, Leoutsakos JS, Sieber FE, Joshi D, Wanamaker BL, Rios-Robles J, et al. Evaluation of two delirium screening tools for detecting post-operative delirium in the elderly. Br J Anaesth. 2013 Oct;111(4):612-8.

44 Hargrave A, Bastiaens J, Bourgeois JA, Neuhaus J, Josephson SA, Chinn J, et al. Validation of a nurse-based delirium-screening tool for hospitalized patients. Psychosomatics. 2017 Nov - Dec;58(6):594-603. 